Quantitative-other

\section{Nepali women who are continuously supported by their husbands during labour are more likely to report greater postnatal support and lower levels of anxiety}

10.1136/eb-2013-101676

\section{Lesley Dixon}

Practice Advice and Research, New Zealand College of Midwives, Christchurch, New Zealand

Correspondence to: Dr Lesley Dixon, Practice Advice and Research, New Zealand College of Midwives, 376 Manchester St, Christchurch 8014, New Zealand; practice@ nzcom.org.nz

Commentary on: Sapkota S, Kobayashi T, Takase M. Impact on perceived postnatal support, maternal anxiety and symptoms of depression in new mothers in Nepal when their husbands provide continuous support during labour. Midwifery 2013;29:1264-71.

\section{Implications for practice and research}

- The transition to parenting involves major changes for most women and support is often needed.

- Maternity caregivers need to assess sources of social support for each woman and promote understanding within her community of the importance of social and physical support during the postnatal period.

- The influence of emotional, physical and psychological support on a woman's psychosocial health following birth continues to be poorly understood and requires more research.

\section{Context}

The transition to motherhood is a major life change which requires a period of adjustment for most women. This change can affect a woman's personal identity and social role, and lead to anxiety and increased risk of depression in the weeks and months following birth. ${ }^{1}$ There is a growing body of evidence linking postnatal depression with adverse effects on the developing infant. ${ }^{2}$ Sapkota and colleagues describe a research project conducted in Nepal in which husbands were present and provided support to their wives during labour and birth. The purpose of this study was to determine whether continuous labour support had an ongoing effect on the mother's perception of support during the postnatal period.

\section{Methods}

The study was undertaken in a referral hospital in Kathmandu, Nepal with low-risk primigravida women approached when admitted during labour. Women were allocated to one of the three groups-those who received continuous labour support from their husbands, those who received support from a female friend and those who did not receive continuous support. Three tools were used to determine postnatal support and symptoms of depression: the Edinburgh Postnatal Depressive Scale, the State-trait Anxiety Inventory for adults (STAI) and the Postpartum Support Questionnaire. These were administered when the woman returned to the postnatal clinic at 6-8 weeks after the birth, or by telephone.

\section{Findings}

Women who received support from their husbands during labour perceived greater levels of support during the postnatal period compared with women who had not received labour support. Additionally, they were less likely to experience anxiety and had fewer symptoms of depression. Women who received support from a female friend during childbirth also reported a higher level of support during the postnatal period compared with unsupported women. An important finding was that the presence of female support at home during the postnatal period resulted in greater perceptions of support, which was enhanced when women had also received continuous support from their husbands during labour and birth.

\section{Commentary}

Historically, women gained support from each other during labour and the transition to motherhood. During the 20th century, birth moved into hospital in many industrialised societies and hospital policies dominated care provision. Attendance at birth was controlled by the institution, resulting in a curtailment of support for women from family and friends. This led to criticism of maternity services and a movement to enable the attendance of partners at birth, a situation which is now the norm in many countries. The involvement of fathers at birth and in parenting has subsequently been found to be beneficial for the health and welfare of the mother and the baby. It is interesting to see how Nepal has followed these trends (albeit many years later) with the move to hospital birth and limited access, involvement and support from partners.

This paper documents the initiation of support for women during labour from partners or a female friend in one of the maternity hospital in Nepal. This research is vitally important for women and maternity health practitioners in Nepal, as it confirms the benefits of support during labour from partners and friends. However, by also looking at the ongoing impact of continuous labour support on the postnatal period, this paper also recognises the fundamental role that support plays in a woman's transition to parenthood. Adaptation to motherhood involves physical, emotional and psychological changes which may be positively affected by social support. In this study, women who were supported during the postnatal period had less anxiety and symptoms of depression, an outcome which underlines the importance of this social support and its influence on maternal mental health. The key message is that for a successful transition to parenting, women need to be well supportedduring childbirth and the postnatal period.

Competing interests None.

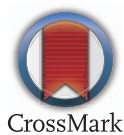

\section{References}

1. Darvill R, Skirton H, Farrand P. Psychological factors that impact on women's experiences of furst-time motherhood: a qualitative study of the transition. Midwifery 2010;26:357-66.

2. Weikum W, Mayes L, Grunau R, et al. The impact of prenatal serotonin reutake inhibitor (SRI) antidepressant exposure and maternal mood on mother-infant interactions at 3 months of age. Infant Behav De 2013;36:485-93. 